\title{
Supporting Real-Time Operations and Execution through Timeline and Scheduling Aids
}

\author{
Jessica J. Marquez ${ }^{*}$ and Guy Pyrzak ${ }^{\dagger}$ \\ NASA Ames Research Center, Moffett Field, CA, 94035 \\ Sam Hashemi ${ }^{\ddagger}$, Samia Ahmed ${ }^{\S}$, Kevin McMillin**, and Joseph Medwid ${ }^{\dagger \dagger}$ \\ San Jose State University Research Foundation, San Jose, CA, 95192 \\ Diana Chen ${ }^{+}$and Esten Hurtle ${ }^{\S \S}$ \\ Carnegie Mellon University, Pittsburgh, PA, 15213
}

\begin{abstract}
Since 2003, the NASA Ames Research Center has been actively involved in researching and advancing the state-of-the-art of planning and scheduling tools for NASA mission operations. Our planning toolkit SPIFe (Scheduling and Planning Interface for Exploration) has supported a variety of missions and field tests, scheduling activities for Mars rovers as well as crew on-board International Space Station and NASA earth analogs. The scheduled plan is the integration of all the activities for the day/s. In turn, the agents (rovers, landers, spaceships, crew) execute from this schedule while the mission support team members (e.g., flight controllers) follow the schedule during execution. Over the last couple of years, our team has begun to research and validate methods that will better support users during realtime operations and execution of scheduled activities. Our team utilizes human-computer interaction principles to research user needs, identify workflow processes, prototype software aids, and user test these. This paper discusses three specific prototypes developed and user tested to support real-time operations: Score Mobile, Playbook, and Mobile Assistant for Task Execution (MATE).
\end{abstract}

\section{Nomenclature}

$D$-RATS $=$ Desert Research and Technologies Studies

$I S S=$ International Space Operations

MATE $=$ Mobile Assistant for Task Execution

$M M C C=$ Mobile Mission Command Center

NEEMO = NASA's Extreme Environment Mission Operations

PLRP = Pavilion Lake Research Program

$P S I=$ Phoenix Scheduling Interface

SPIFe $=$ Scheduling and Planning Interface for Exploration

\section{Introduction}

$\mathrm{N}$ ASA Ames Human Computer Interaction Group, for the last ten years, has developed scheduling and planning software tools that support the operations of a diverse set of space missions. We call our scheduling and

\footnotetext{
${ }^{*}$ Human Systems Integration Division, NASA Ames Research Center, M/S 262-2, Moffett Field, CA 94035, and AIAA Member.

${ }^{\dagger}$ Human Systems Integration Division, NASA Ames Research Center, Moffett Field, CA 94035.

${ }^{\ddagger}$ Human Systems Integration Division, NASA Ames Research Center, Moffett Field, CA 94035.

${ }^{\S}$ Human Systems Integration Division, NASA Ames Research Center, Moffett Field, CA 94035.

${ }^{* *}$ Human Systems Integration Division, NASA Ames Research Center, Moffett Field, CA 94035.

${ }^{\dagger \dagger}$ Human Systems Integration Division, NASA Ames Research Center, Moffett Field, CA 94035.

\# Human Computer Interaction Institute, Carnegie Mellon University, Pittsburgh, PA 15213.

${ }^{\S}$ Human Computer Interaction Institute, Carnegie Mellon University, Pittsburgh, PA 15213.
} 
planning software toolkit SPIFe (Scheduling and Planning Interface for Exploration, pronounced "spiffy"). For each space mission, a customized version of SPIFe has been deployed for operations ${ }^{1}$. For instance, the Phoenix Scheduling Interface (PSI) was developed and tailored by our group for the Mars Phoenix mission. Similarly, other Mars missions (Mars Exploration Rovers, Mars Surface Laboratory) are using versions of SPIFe as part of their daily surface operations. While the legacy of SPIFe is rooted in robotic missions ${ }^{2}$, different instantiations of our software have recently been deployed to conduct daily operations for International Space Station.

Much of our work has focused on supporting the task of scheduling and planning, which in the space domain is a complex, constrained problem. Our end-users, be it scientists, engineers, or flight controllers, complete this task in a timely manner, varying from a few hours to a few days. SPIFe, in general, provides a method of defining activities in time alongside an interface and visualization for the collection of activities to be accomplished. Our software toolkit has a lot of functionality that facilitates this task, like for example, helping users identify and resolve violated constraints that exist in a proposed plan. Many SPIFe users have requested additional aids that would help them follow-along the scheduled plan in real-time within the software application itself ${ }^{3}$.

However, through user observations, we have learned that this strategy, while useful in providing real-time aids on a desktop application, limits the number of end-users that could actually use and view the scheduled plan. This paper discusses the multiple prototypes we have developed, assessed, and field tested in the last couple of years, aimed at providing real-time execution software tools to a broader set of users. We briefly provide an overview of current aids that exist in the domain of human space operations, followed by our experience with Score Mobile, Playbook, and Mobile Assistant for Task Execution (MATE).

\section{What is in a Plan?}

\section{A. Planning and Schedule for Human Space Operations}

Flight controllers plan for all aspects of mission operations for the International Space Station (ISS). As an orbiting, complex spacecraft, its operations require constant planning in order to support the six crewmembers that live on-board. The focus of this paper is on the plans created for these astronauts, plans that are shared with the flight controller community, and finally, extensively used on-board the ISS. Astronauts have their schedule carefully planned based on a large set of operational constraints and objectives set forth by the space program. Some of the operational constraints (which are also highly interrelated) include communication availability, accessible power resources, and the spacecraft's position. Furthermore, activities have to be coordinated with all the other international partners that help manage the space station. During their six-month long stay on ISS, crewmembers must maintain the space station and complete a

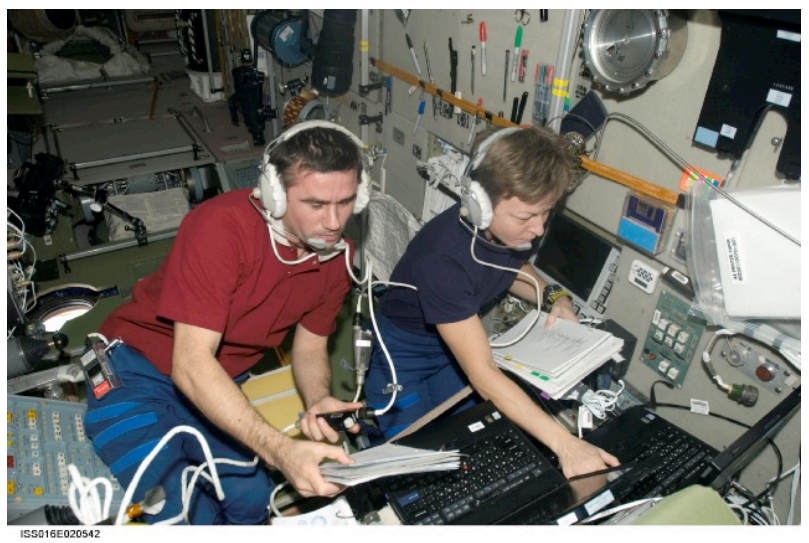

Figure 1: ISS astronauts collaborating and completing an assigned activity

large, diverse set of research experiments. Despite seeing the sun rise every 90-minutes, astronaut sustain a sense of daily routine, which includes coordinated meal times, night shifts, and extensive exercise routines to counter the debilitating effects of long-term effects of microgravity on their bodies.

The essence of ISS plan for astronauts is relatively straight forward: individual crewmembers have a set of activities that must be completed each day. Activities are assigned to one or more astronauts (Figure 1). These activities can be:

- routine, e.g., daily conference with ground,

- periodic, e.g., replacing worn mechanisms in exercise devices,

- event-driven, e.g., removing stowage from visiting spacecraft, or

- mission-specific, e.g., scheduled research experiments or maintenance spacewalks.

Often, an activity is to be completed by more than one crewmember, requiring astronauts to collaborate. Other activities require cooperation of ground controllers, necessitating voice and video communications. Some activities can only be done by one crewmember at a time (like for example, using a particular workstation). Coordinating and synchronization of multiple schedules is intricate, but essential for ISS operations.

2

American Institute of Aeronautics and Astronautics 
Most of the activities scheduled for ISS have associated procedures and instructions for crewmembers. Astronauts need specific instruction on how to perform the tasks assigned to them. Unlike spacewalks, most intravehicular activities are not repeatedly practiced and synchronized to the minute. Astronauts are given skill-based training to execute most maintenance tasks and background science training for specific research experiments. However, for many non-routine activities, astronauts refer and depend on procedures. The procedures are step-bystep instructions to complete tasks, often with specific notes (referred to as execution and ops notes) associated with that day's activity. Additionally, many activities have stowage "notes" associated with them, which is a list of all items and their location in ISS that are necessary to complete the given task.

While the plan itself may appear to be simple, it is developed over many weeks of coordination, careful planning and scheduling, resulting in a plan that abides to the operational and programmatic constraints. Once a schedule is agreed upon all the international partners as well as two NASA centers, any change to the plan must be reviewed and approved by the appropriate set of flight controllers. Changes can be as large as reassignments or additional activities to as simple as updates to procedures or execution notes.

Once the plan "goes live," i.e., the day of execution, everyone from crew to flight controllers view the schedule. Viewing the schedule is a critical part of daily ISS operations. Crew access the timeline and associated procedures in order to execute all their assigned tasks in a timely manner, respecting the scheduled constraints. The ground follows along the schedule, not only to keep situational awareness of what is occurring in the space station, but also if needed for coordinated activities. Crew provides feedback on the timeline as to their progress and completion of tasks, which in turn flight controllers access and use as a means of collecting data about the scheduled activity.

\section{Visual Layout of a Plan}

Currently, ISS mission operations is updating their scheduling and planning software tools. Score (a version of SPIFe for ISS crew planning, Figure 2) supports the planning phase, during which flight controllers schedule activities for six astronauts. Once a plan is completed, it is used and seen by crew and flight controllers via a web viewer interface. The viewer is a separate software tool (i.e., not part of Score); other web aids are used to connect activities to their associated procedure.

Figure 2 depicts the typical visual layout of a schedule. This layout is unusual when compared to common calendar arrangements, though perhaps not so different to program management scheduling. Each crewmember has a "band", a horizontal layout of each scheduled activity. All crewmembers' schedule are stacked, allowing for simultaneous viewing of everyone's activities at any one point in time. Similarly, there are horizontal "bands" for important conditions, such as when ISS is scheduled to be in daylight or when voice communication is available. The use of color is driven by various operational reasons, including depicting groupings of activities or the need to complete that activity at precisely the designated time.

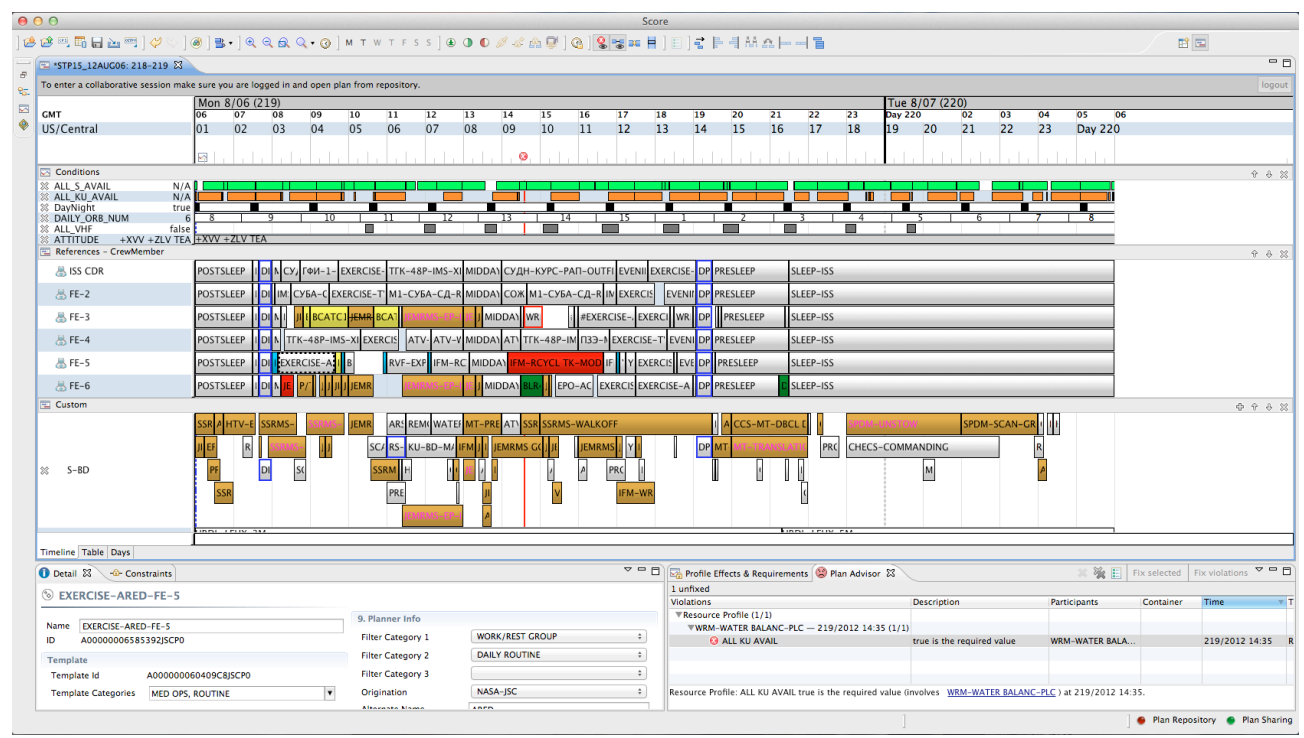

Figure 2: Score user interface, with a day's schedule for six astronauts 


\section{B. Earth-Analogs for Human Space Exploration}

The NASA Ames HCI Group has contributed to advancing the state of the art of scheduling and planning, and we have started researching how to improve upon supporting real-time operations and execution of planned schedules. In order to investigate and collect data on the best methods to support real-time execution, we have strategically conducted user testing and field tested software prototypes of timeline viewers to Earth-analog sites.

Earth-analogs are simulated exploration missions organized by NASA (or NASA-sponsored) aimed at investigating different aspects of deep-space human exploration. Score has been used as the planning and scheduling tool for a variety of these earth-analog missions. We have also had the opportunity to conduct research at the following analogs: Pavilion Lake Research Program (PLRP), Desert Research and Technologies Studies (RATS), and NASA's Extreme Environment Mission Operations (NEEMO).

Each of these analogs emphasizes a different aspect of exploration to simulate, and typically, their "crewmembers" are mostly composed of current astronauts. In PLRP, the focus is scientific exploration in an extreme environment. Scientists use a combination of divers, piloted/personal submarines, and remote control underwater robots to explore lakes in British Columbia in search of freshwater microbialites. Desert RATS emphasizes field testing new technologies and mission operations strategies, in the context of planetary habitats and a variety of transportation architectures, from rovers to asteroid-grappling spacecrafts. NEEMO, having an underwater habitat, allows for six crewmembers to live in a confined space for two weeks while completing a series of research experiments. For all analogs, a daily schedule must be defined and executed, and all team members view and share the same timeline.

\section{Score Mobile}

\section{A. Driving User Needs}

Our data collection and user observations started during the 2010 analog season, where we observed crew members and support members of analogs having a difficult time following along with the current and upcoming activities scheduled for the current day. The integrated activity plan, developed by the mission planners, was only available in Score, which was only installed on the workstations located in the crew's habitat and on workstations in the Mobile Mission Command Center (MMCC). Additionally, easy access to these workstations was not feasible. Often, crew members were executing activities or moving between locations to perform their activities. While away from a workstation, analog crewmembers were not aware of how much time was being spent completing the current activity or how soon the next activity would start. As a result, crewmembers had to the additional burden of returning to a workstation or use communication channels to ask planners in the MMCC about the plan. Both of these methods disrupted the completion of tasks, potentially impacting the rest of the activities scheduled for the day.

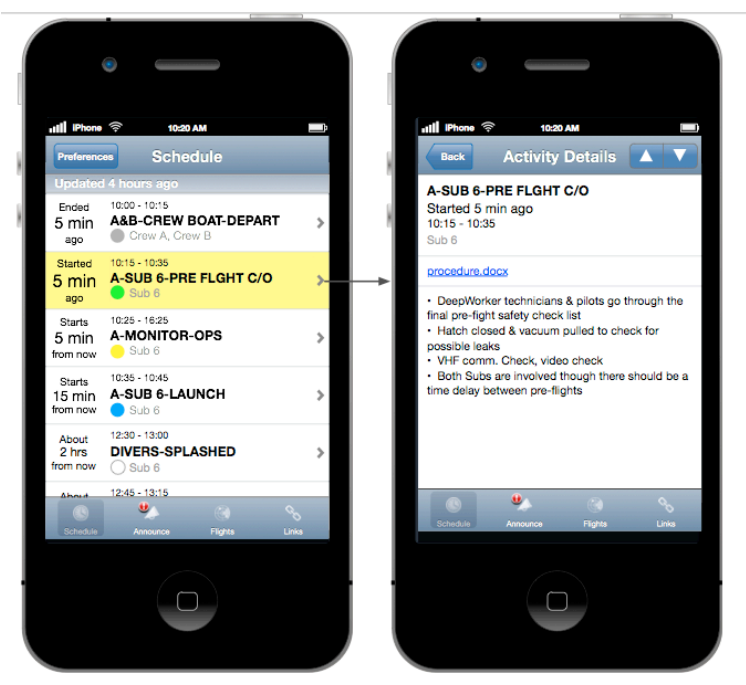

Figure 3: Score Mobile interface design

These observations led to the first driving user need that has guided the rest of our research: the need for a mobile, light-weight viewer. Score, being a desktop application, is not suited nor designed to currently support this need, hence, Score Mobile was developed. The primary goal of Score Mobile was to give crew and the analog support team members access to upcoming activities scheduled for the day through their existing smart phones and mobile devices (such as tablets). A walk-up-and-use interface was designed to fit the needs of analog team members, allowing them to quickly peruse the plan and understand what activities were currently occurring as long they had internet access.

\section{B. Prototype Capabilities}

The Score Mobile development team utilized the emerging mobile web framework, JQuery Mobile and the Score client to quickly develop a web application. Score Mobile was used in three space analogs in 2011, PLRP, Desert RATS, and NEEMO. For each subsequent analog, Score Mobile was adapted for the specific needs and improved based on observation and user feedback. 
The initial release displayed activities from the plan in chronological order in a vertical list (Figure 3). Throughout the day, the list updated to show the current activities at the top highlighted in yellow. Users could pick from several timeline bands to customize the display. Each timeline band contained the activities for a single crew member or an analog support team. An overview timeline band was also created to allow team members to understand the general themes of the day. Upcoming activities showed a count-down timer, indicating the amount of time until the next activity. Users could find further information about activities' details as well as access a PDF copy of the entire schedule. Score Mobile also displayed notifications to the whole team which served as a simple group text message system, including notifications for when a new, updated version of the day's plan had been uploaded.

Score Mobile was first deployed in the Pavilion Lake Research Project (PLRP) analog. Team members used the mobile website to follow the activity plan, check on submarine flight paths (embedded map images), and share announcements with team members. We learned many important lessons from this analog and collected feedback from the support teams. One of the critical lesson was that crewmembers wanted more than just the basic details of an activity; crewmembers also wanted access to the execution procedures, which were not linked through Score Mobile. This additional capability was provided to Score Mobile in support of the Desert RATS campaign (Figure 4).
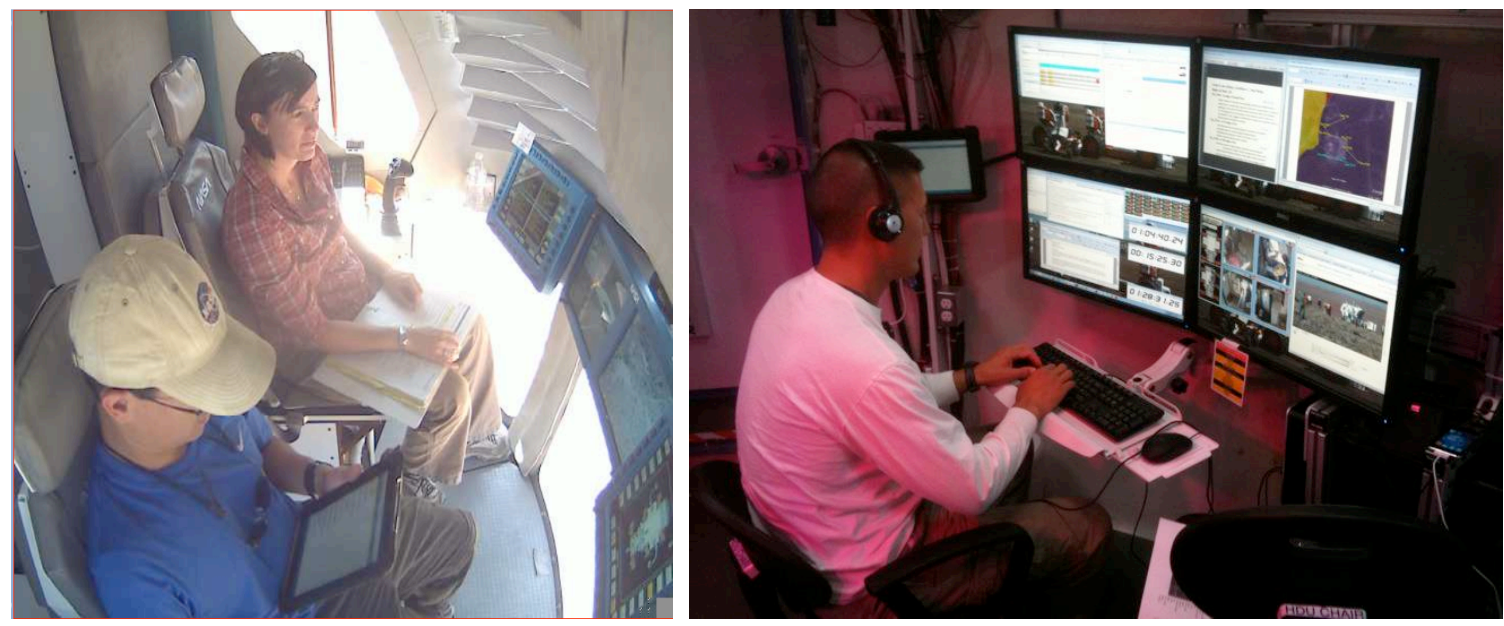

Figure 4: Desert RATS crewmembers using Score Mobile in the rover and inside habitat

Post-analog questionnaires were completed by crewmembers, providing us with Score Mobile feedback. An unexpected finding was that Score Mobile was also used by crew members to review upcoming plans during offhours. Crew members appreciated the ease of use of Score Mobile and the fast access to the PDF timeline available in their mobile devices. One important feedback indicated that improvements could be made to better illustrate durations of activities. In Score Mobile, the vertical layout of a scheduled depicted a 5 minute activities in the same way as 5 hour activity. Users could not quickly assess the duration or the time left in an activity by just glancing at the timeline. Additionally, users wanted more control over which activities were focused on. When an activity took longer than expected, the activity would scroll off the page, crew members were unable to change the plan or change the status of activities to indicate they were taking longer than expected.

Another key feedback provided by the RATS support operations team and crewmembers was the value of seeing the "as run" plan in real-time. This particular feature was implemented for the NEEMO analog, making it the primary interface for both the support team and the crewmembers. Score Mobile was integrated into Score to allow support teams to view the as run plan alongside upcoming changes to the plan,. Planners were given a "post to Score Mobile" shortcut button in Score, permitting quick updates of the plan to the website throughout the day, such that team members would always get the

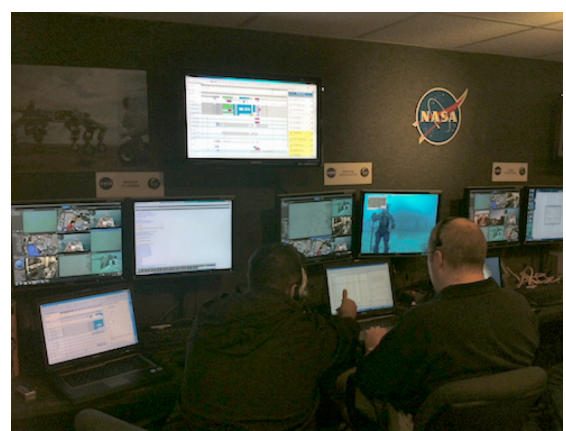

Figure 5: Score Mobile in NEEMO's MMCC 
latest version of the plan. Score Mobile was displayed and used by crewmembers who were in the underwater habitat and by the support team in MMCC (Figure 5). We received similar feedback with respect to ease of use, but an important, additional feedback provided by the users of Score Mobile at NEEMO was that crew wanted to also easily communicate plan updates to the surface team.

\section{Playbook}

One essential data collected during the 2011 analog season was the need to support real-time crew feedback through Score Mobile. Most of our efforts had focused on pushing the planned schedule out to various, diverse set of users, but we did not consider nor facilitate new scheduling information being pushed back to the mission planners. Unfortunately, the developed infrastructure of Score Mobile did not easily allow for that capability. A new mobile web application was needed, and hence, Playbook was developed. Designed and developed from the data collected from previous analog seasons, incorporating lessons learned from Score Mobile, Playbook would serve as a central location for crew members to access plan, and procedure data, as well as allow the crew to communicate with the rest of the analog team.

\section{A. Driving User Needs}

Like Score Mobile, Playbook was built as a situational awareness tool for both crew and support teams. Building upon the positive feedback and the needs identified by analog crewmembers as well as support teams, Playbook design and implementation focused on the following five key needs:

1. Tablet-focused interactions. In analogs, crew had shown a strong preference for using iPads to view schedule and procedures. Interviews with ISS crew had shown similar requests. Score Mobile had been designed primarily for use on phones, which meant limiting the amount of information on the screen. A design aimed at tablets needed to be much richer, showing items like multiple individuals' schedules on the screen. While we wanted to address the platform preference, we also knew that Playbook must scale to a variety of devices, as the support teams preferred using laptops and phones.

2. A visual timeline. Score Mobile feedback indicated that durations of

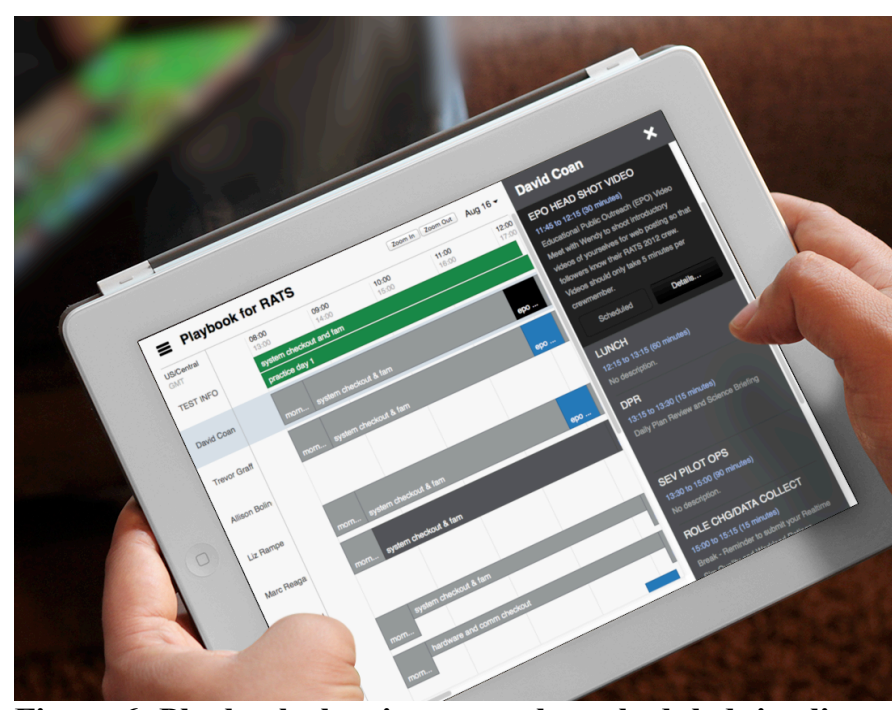

Figure 6: Playbook showing an analog scheduled timeline current and future activities was still an important piece of visual information that this prototype lacked. Users asked for a timeline view that showed all activities happening in parallel, akin to the visual presentation in Score (Figure 2) and current ISS schedule viewers. A shift to tablet-focus platforms provided us with the ability to include this feature.

3. Ability to edit the schedule. In dealing with complex schedules, analog crew quickly discovered the plans would change on-the-fly. To increase situational awareness, and to make sure everyone was on the same page, crew requested the ability to edit the schedule -- indicating status changes to activities (in progress, complete, aborted, etc) or rescheduling the activities to new times.

4. Live Updating. One of the key findings from Score Mobile was the importance of keeping everyone involved in the mission on the same page. Playbook needed to allow for the above capabilities while constantly updating to any changes made by support teams. When this was not possible, such as in adverse network conditions, it was important to notify people that their information was out of date.

5. Embedded procedures. Originally, Score Mobile was not designed as an execution aid. Improvements were made to Score Mobile to address this but Playbook was designed with the idea of increasing crew efficiency by imbedding materials required for execution within the schedule itself. To increase crew 
efficiency, procedures and supporting documents such as stowage notes need to be quickly accessible, ideally embedded within the schedule itself.

\section{B. Prototype Capabilities}

Playbook shows a full timeline view, showing bands for each user and any supporting role (Figure 6). The schedule is imported from Score, easily refreshed, and reflects the schedule that was carefully constructed by the ops planner. This tightly-knit connection is essential, allowing the ops planners to have control of the schedule and data the rest of the analog team views. By default, the view shows a single day at a time. The "day picker" shortcut (in the top right) can be used to switch between days. The zoom buttons can alter the amount of information shown and on touch-screen devices, an intuitive pinch and zoom gesture can be used as well.

Tapping an activity opens an area on the side called "the stream" (Figure 6). This is a focused view of a single band. Depending on how a band was defined, it can show all the activities associated with a person, place, or piece of equipment. Selecting one of these activities in the stream puts it into focus. Each activity can then be updated by the user, for instance, updating the activity's status or add notes to the activity. Equally, tapping individual activities in the timeline allows users to update them. This two-way selection is important -- allowing users to quickly switch between seeing the details of an activity and seeing how that activity fits into the bigger context of the mission.

Once an activity is selected, any associated procedures appear inline. Selecting the name of the procedures opens a new browser tab with a detailed step by step walkthrough of the procedure. This "new tab" behavior allows multiple procedures to be opened at the same time, permitting crew to reference multiple documents, if necessary, in complex situations.

In addition to the schedule, other information is available through the master navigation toolbar (Figure 7). The "Procedures" section is a focused list of all the procedures across the activities. The "Mission Log" evolved over the course of several analogs. In early iterations, the mission log was a running log of all the changes happening in the mission. Users in the NEEMO analog found this useful, but overwhelming: the log would quickly fill with crew marking activities as "complete". Notes and aborted activities would get buried. The concept was revamped to be an off-band communication channel with rich media (Figure 8). Users could send a picture, video, or text note and it would automatically appear on everyone's screen without interrupting the on-going voice loops. In the RATS analog this proved to be especially useful in sending science updates, e.g. new traverse maps for the crew.
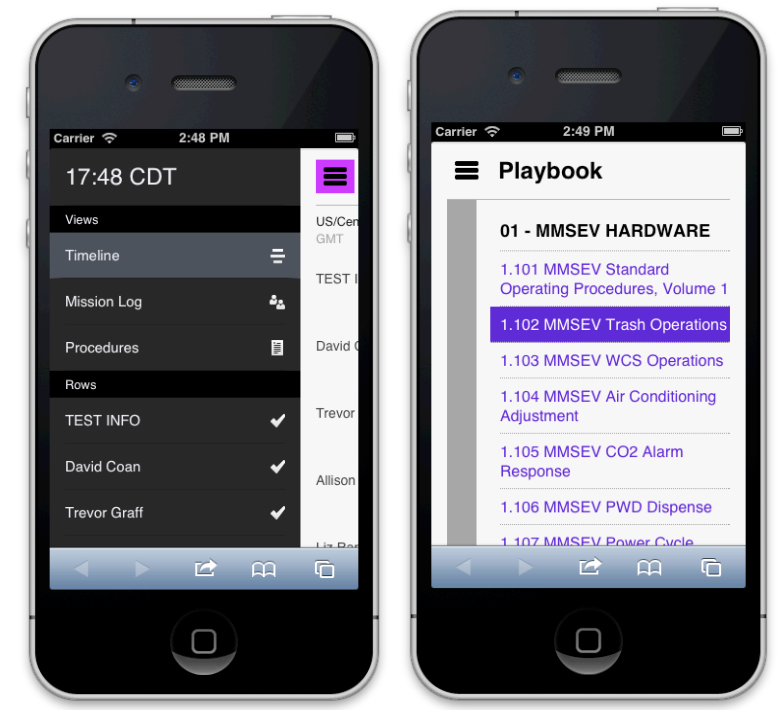

Figure 7: Playbook as viewed in on mobile device. Navigation toolbar shown on left, procedure accessibility shown on right.

\section{Future Work}

Playbook was used in 4 space analogs in 2012, with continuing refinement between each. We received positive feedback from analog crewmembers, who described Playbook as highly useful to their day-to-day operations. Some comments included: "Mission enhancing tool -- essential" and "Everything you need in a quick to touch format." As 
in our previous experience in analogs, crewmembers and analog support teams suggested improvements. Crew selfscheduling is still a desired functionality. Early prototypes of Playbook have begun to explore this by introducing the ability to highlight activities that can be moved without resource impacts, allow users to drag them around, and send the new schedule back to ground. Future work will look at allowing larger degrees of flexibility and doing live resource calculations to show the exact impact of changes.

Our experience in earth-analogs and feedback provided by crewmembers indicate that rich, interactive procedures is a foreseen, natural progression to current established procedures. Current procedure technology is reaching its limits -- crew must follow long-written descriptions of procedures steps and have an especially difficult time jumping between procedures. To deal with the former, Playbook has tested embedding videos and audio directly into procedures, potentially simplifying procedures for crewmembers. In the future, Playbook may introduce and support the concept of "compose-able" procedures, where multiple procedures are embedded together on a single page to form a master procedure, eliminating the need to jump between disparate files. Some additional improvements in this area include automatic timestamp logging and integrated telemetry.

In the same vein, multimedia in communication loops seems to be desired future functionality, particularly in mission architectures where communication is conducted in an asynchronous manner, like it was in 2012 RATS Earth-analog. The acceptance and positive feedback received on the "mission log" used at the RATS analog validates the need for an asynchronous communication channel that includes a variety of inputs, whether it be video, audio, or text. This notion of a "mission log" is not foreign to mission operations, as flight controllers monitor communication voice loops. Future mission logs could be akin to multimedia communication loops. Including this capability in Playbook will better serve analogs that are simulating future, long duration and long distance exploration missions.

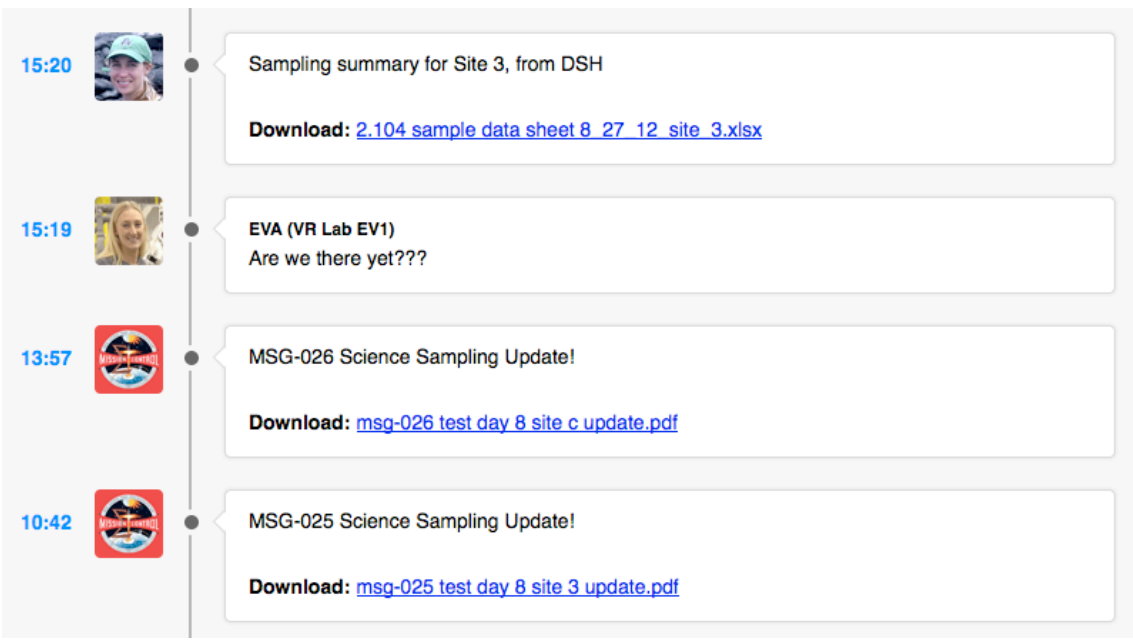

Figure 8: Playbook's Mission Log

\section{MATE}

\section{A. Driving User Needs}

To explore mobile-focused software to support operations execution, the HCI Group mentored a student research and prototyping project with Carnegie Mellon University. The result was a capstone project and the development of the Mobile Assistant for Task Execution, or MATE, which focused on supporting complex task execution through procedures within the context of asynchronous communications for space operations. The MATE research is different from Score Mobile and Playbook as we were able to research analogous domains, derive lessons learned from these, and investigate their application to the space domain through user testing. We observed and/or interviewed automotive technicians, surgeons, construction managers, and theatrical stage managers, in addition to NASA-associated end-users, including former astronauts and crew members of the NEEMO program. Based on this research, we focused on a few key findings from which three primary design objectives emerged for MATE: provide intuitive support aids for learn-ability, allow users the flexibility to handle interruptions, and present all data associated with a procedure in one, minimally-cluttered screen. 


\section{B. Prototype Capabilities}

MATE prototype has four key features: the crewmember's list of daily activities (the "home view"), a dedicated activity view for each activity (the "activity view"), persistent note-taking area, and a ground communication panel. The home view gives an overview of the crewmember's day as well as relevant contextual information, including recent notes, scheduled lapses in communication, and the most recent daily planning summary. The activity view provides the user with all of the information needed to execute a scheduled activity. Features such as progressmarking and step folding help reduce cognitive load during execution, while a built-in timer helps the crewmember stay on schedule without feeling rushed. The note-taking interface lets users create notes for long-term communication as well as make them available for later use. The notes could be at associated with the whole day or comment can be connected to just a specific activity step. Finally, ground communication was implemented through a text-based message center, permitting crewmembers and ground crew to communicate with one another asynchronously. Non-intrusive visual notifications informed users of incoming messages.

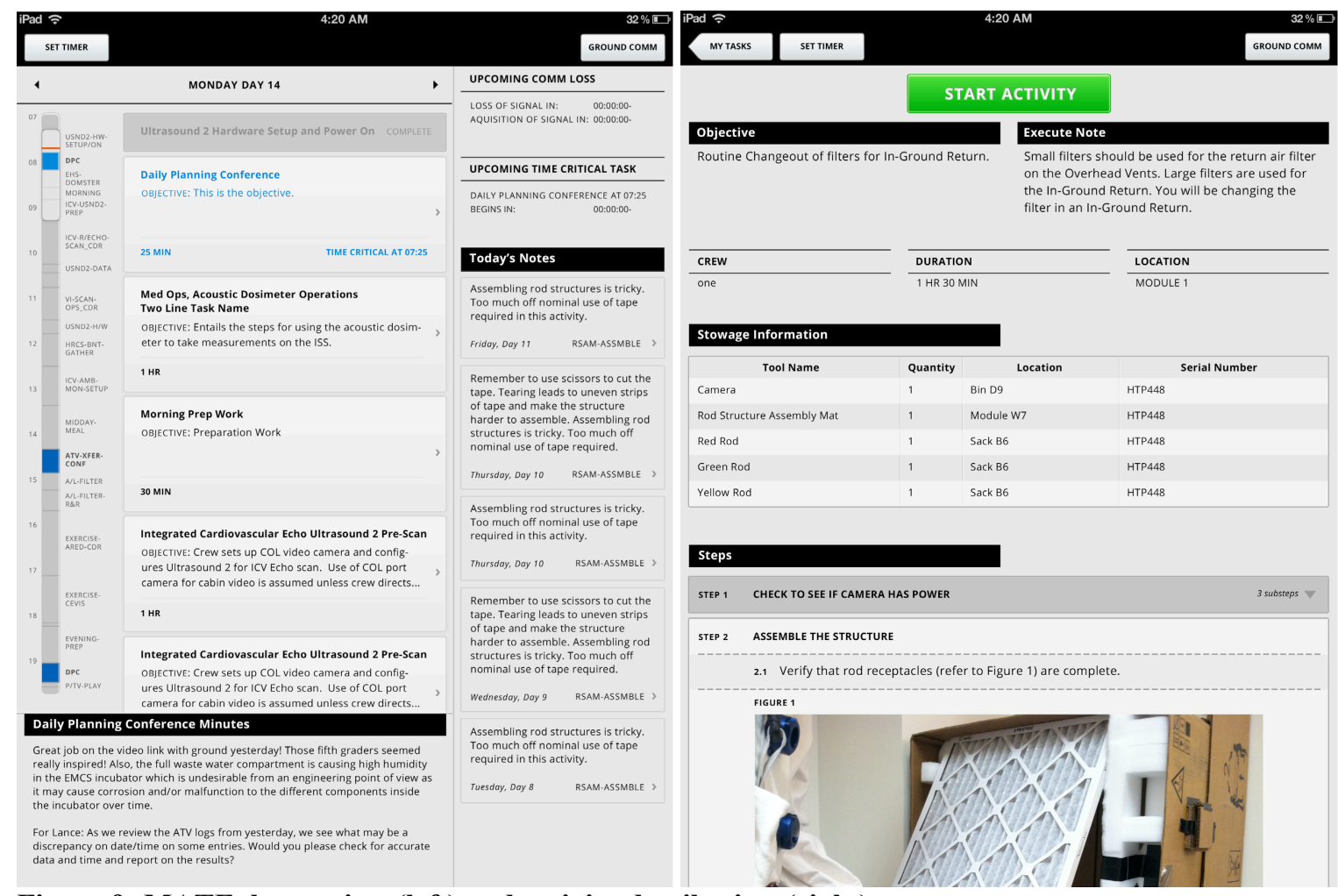

Figure 9: MATE, home view (left) and activity details view (right)

\section{User Testing Results}

MATE went through five prototypes iterations, each with increasing fidelity (i.e., from paper to interactive PDF to iPad application). Usability testing at each round provided foci for the next iteration. Early tests were aimed at creating a visual language for displaying the steps involved in task execution. These initial tests led to features specifically aimed at maintaining visual clarity, such as progress tracking and a communication log that hides itself when not needed.

By having users conduct activities of increasing complexity throughout development, MATE was able to communicate the complex, information-rich instructions (ISS-like procedures) without cognitive overload. We were able to demonstrate this by recruiting naïve participants and asking them to use MATE after a short ten-minute training session. These users were asked to complete a nonlinear series of objectives designed to exercise implemented features, giving the user the freedom to transition between tasks and interface tools as they saw fit (Figure 10). These scenarios tested MATE's core features, each designed to be as intuitive as possible to reduce cognitive load in high-stress, interruption-prone environments. Test participants exhibited near-perfect performance (with respect to completing the assigned tasks), despite multiple interruptions and shifts in procedure context. This 


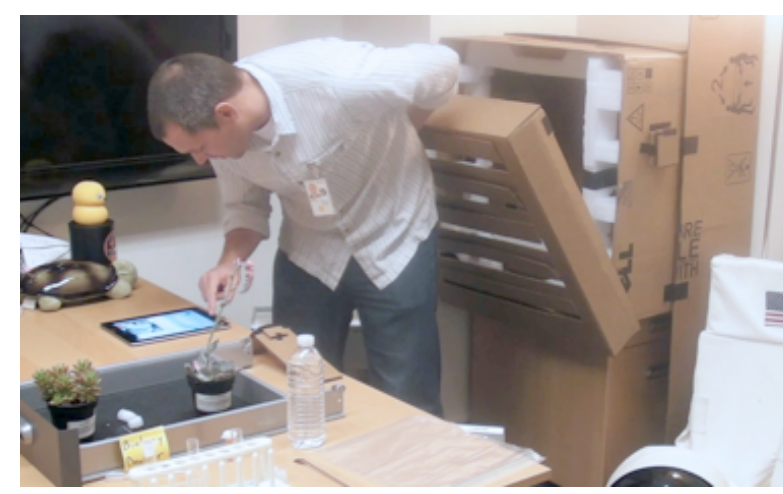

Figure 10: Participant executing complex task using MATE combination of familiarity and simplicity also allowed users to use MATE in unexpected ways, developing unique workflows and strategies in situ.

Due to its limited development cycle, MATE ultimately did not include every feature that would be desirable for task execution onboard the ISS. Note-taking with rich media, for instance, was high on every user's list of desired features but was deemed too technically difficult for this prototype. Issues also arose with user compliance, specifically in the area of progress tracking. Despite these areas not fully explored, MATE was a fundamental success in its ability to enable the essential communication and task execution needed to conduct complex procedures in space.

\section{Concluding Remarks}

Field-testing software prototypes in Earth-analogs has permitted exploration of new ways of providing support for real-time space mission operations and execution of scheduled activities that currently are not in place for ISS operations. Our research experience shows that mobile devices have become an essential component to sharing and viewing schedules among a large team. This conclusion is not too surprising considering how ubiquitous mobile devices are now-a-days. Furthermore, multiple types of mobile platforms (e.g., phones, tablets, laptops) have to be supported as various end-users have different needs. Our research also indicates that it is essential to design schedule viewers for multiple mobile platforms from the beginning, as retrospectively incorporating this capability is technically challenging and results in missing functionality.

One design challenge we constantly had to address for with our real-time execution prototypes was the need to implement a software aid that was easy to "pick up and use." For Score Mobile, Playbook, and MATE, there was minimal to no training for these tools, yet they had to serve the needs of a very diverse set of end users. One set of users wanted to a quick, easy method of obtain the necessary situation awareness of current and future activities. Aside from simply viewing the timeline, most of these users have expectations of receiving summary information, as in a "feed". All of our prototypes supported this, be it through an overview "band", the mission logs, or the asynchronous text updates from "ground".

Another set of end users, namely, crewmembers, aside from wanting a mobile viewer and needing situation awareness of the overall day's schedule, also require much more detailed information about the day's activities. Much of our research focused on learning about their needs, as these users are different from the typical mission operations planners that operate SPIFe. User tests of Score Mobile, Playbook, and MATE has shown that crewmembers need software aids to support the following three aspects of real-time execution: how to complete a task, how long do they have to complete it relative to the rest of the schedule, and how to communicate to others progress. Moreover, these aspects should be tightly knitted together in their design and implementation in order to be effective. In every prototype described, we tried to better integrate procedures into activities, evolving with each iteration. One aspect which we can improve upon still is providing crewmembers better indications of progress and remaining duration, ideally combining these with progress or notes on procedures and in turn, automatically communicating this to ground controllers.

Finally, with regards to supporting real-time communication, be it between crew to ground, or amongst flight controllers, the need to support a diverse set of communication protocols was prevalent. Texting or chatting is just one simple addition to the traditional voice communication. However, now-a-days, using multimedia, i.e., video, images, is common, and often requested in the operations of Earth-analog missions. Our prototype tools were quick to adapt and will continue to evolve in order to meet this necessity.

One aspect that we are still working towards in the future is to better support scheduling communications between crew and ground. At a minimum, astronauts want to provide completion updates to flight controllers. Yet, Earth-analog crew request and future long-duration missions will need to support rescheduling requests. Any future real-time operations and execution software aid will have to allow for end-users to request or communicate changes in the planned schedule. One simple example is the use case where a required activity takes longer to complete than scheduled. Astronauts will want to communicate progress and see the impact to the rest of their schedule, requesting changes based on the in situ information they have. Such an aid needs to be tightly integrated with the planning and 
scheduling tool in order to assess if any constraints are not being fulfilled. This work is both a technical and design challenge and easily communicating this information back and forth is not straight-forward.

Over the course of a couple of years, the HCI group has learned much about the needs to support real-time operations and execution of scheduled plans, through mobile devices that allow for situation awareness and activity execution while incorporating flexibility to facilitate various end-users with different types of objectives. Working alongside the operations mission planners in the Earth-analogs has permitted this iterative process of developing and field testing software prototypes with crewmembers and ground controllers. This research allows us to explore the next aspect of scheduling and planning, enabling future software aids for long-duration human space operations.

\section{Acknowledgments}

The authors would like to acknowledge the work, contribution, and support of Mel Ludowise, Jill Foley, Chris Nelson, Michael McCurdy and Don Kalar. Each were valuable assets to at least one of the prototypes described in this paper. NASA Johnson Space Center Mission Operations Directorate supported our Earth-analog research by using Score, Score Mobile, and Playbook. We'd like to thank particularly Marc Spicer, Megan Rosenbaum and Lauren Rush for spear-heading these efforts. We would also like to acknowledge the Human Computer Interaction Institute at Carnegie Mellon University for their continued collaboration with the HCI Group.

\section{References}

${ }^{1}$ McCurdy, M. (2009) "Planning tools for Mars surface operations: Human-Computer Interaction lessons learned” 2009 IEEE Aerospace Conference. Big Sky, MT, March, 2009

${ }^{2}$ Aghevli, A.; Bachmann, A.; Bresina, J.; Greene, K.; Kanefsky, B.; Kurien, J.; McCurdy, M.; Morris, P.; Pyrzak, G.; Ratterman, C.; Vera, A.; and Wragg, S. "Planning Applications for Three Mars Missions with Ensemble." International Workshop on Planning and Scheduling for Space. Baltimore, MD, 2006.

${ }^{3}$ Marquez, J.J., Ludowise, M., McCurdy, M., and Li., J. (2010) "Evolving from Planning and Scheduling to Real-Time Operations Support: Design Challenges". Proceedings of AIAA International Conference of Environmental Systems. Barcelona, Spain, July 2010. 